\title{
Multi-tasking A/D Acquisition System Design and Implementation based on ARM9 Microcontroller
}

\author{
Yutao XIA \\ Weifang University of Science and Technology \\ Shouguang,Weifang,Shandong, 262700 China
}

\begin{abstract}
In order to achieve fast and efficient data acquisition and digital-to-analog conversion based on ARM9 Microcontroller S3C2410 and embedded real-time operating system $\mu$ C/OS-II of multi-tasking A / D acquisition system. Not only able to finish with high precision, reliable, stable, real-time multi-channel analog signals and on-off signal data acquisition, and displays it on the LCD in real time. Completed in ADS1.2 compiled environment, system software using $C$ language and assembly language mix programming, which makes full use of the advantages of different languages. This paper constructs the system's overall design, hardware circuit design method is given in detail, at the same time describes key transplant $\mu$ C/OS-II embedded operating systems and software design.
\end{abstract}

Keywords- ata Acquisition; LCD; Real-time Display

\section{INTRODUCTION}

Data acquisition and processing system based on ARM920T core is most frequently used in the field of singlechip microcomputer application system of the kind. Data capture is the basic means of access to information. The physical data acquisition system for the collection, storage, processing and display, to achieve the monitoring or control of the physical quantity, the processed data sent to the display device for display, can also be sent to another computer through the serial port. With the computer technology in industrial monitoring, control and management of a wide range of applications, data acquisition systems in the industrial automation process played a major role. With the arrival of the post-PC era, embedded systems have been comprehensively penetrated into various aspects such as scientific research, industrial production and daily life, Therefore, the embedded system platform data acquisition system came into being. Embedded systems have portability, easy cutting, system services, interrupt management, stability and reliability advantages. Taking into account the data acquisition system reliability, flexibility and real-time with high demands, the embedded data capture system will have a very broad prospects.

\section{GENERAL DESIGN OF THE SYSTEM}

Data acquisition system mainly complete real-time data sampling, maintain, and is sent to A/D converter into digital signal, and then the data processing, storage, through communication interface sends real-time data to the LCD display. The system based on ARM9 S3C2410 Microcontroller and embedded real-time operating system $\mu \mathrm{C} / \mathrm{OS}$ ii of the two parts of the software. System frame diagram shown in Figure 1.

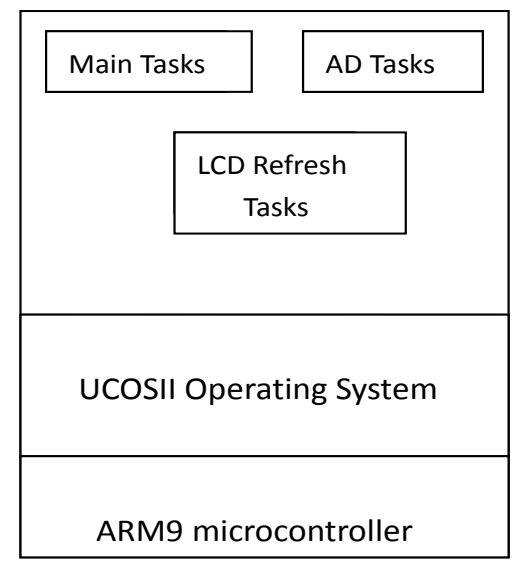

Fig.1. Embedded AD acquisition system block diagram

The top layer is application procedure layer for five tasks, including main_tasks, three AD_tasks and LCD Refresh

Tasks, which priority of each tasks will be set to $12,20,21,22,23$.UCOSII Operating System lies in the middle layer, However, The bottom layer consist of hardware layer which is controlled by ARM9 Kernel to accomplish development for it.

\section{HARDWARE DESIGN}

The system resource is the core of ARM9 MicroController, However, Peripheral circuits mainly contain AD Acquisition Circuit, Power Cicuit( providing voltage for whole module), JTAG Circuit(download procedure),LCD Display Circuit(mainly display the sample data in the form of wave), RS232 Circuit( transmitting data to PC by rs232 protocol).As shown in Figure 2.

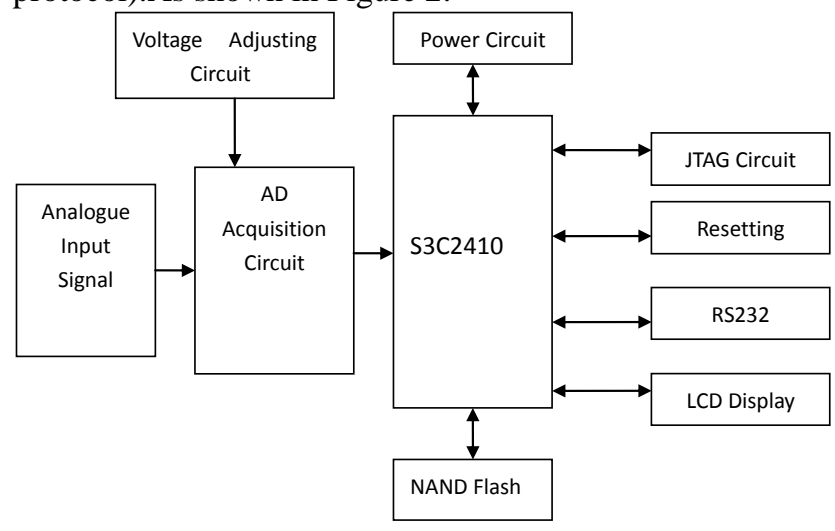

Fig.2. Embedded AD acquisition system block diagram 


\section{ANALOG INPUT CIRCUIT}

S3C2410 has an internal 8-channel 10-bit successive approximation type $\mathrm{A} / \mathrm{D}$ converter, the maximum conversion rate of $500 \mathrm{~K}$. A/D converter maximum clock frequency is 2.5MHz. A/D converter basic clock provided by the APB bus clock, 10-bit accuracy requirements need $11 \mathrm{a} / \mathrm{d}$ conversion clock.

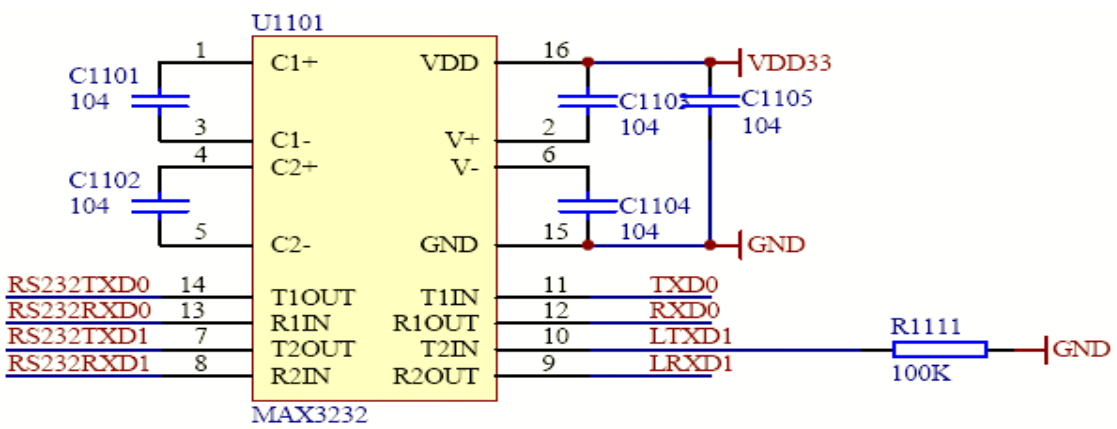

\section{RS232 COMMUNICATION INTERFACE CIRCUIT}

S3C2410 processor comes with three UART ports, each UART channel has 16 bytes of FIFO for receiving and sending. Maximum baud rate for system clock can be up to $230.4 \mathrm{~K}$, if using a UART external clock can run higher baud rate. Therefore, initialing UART1 interface with MAX3232 chip interacts with the PC. Specific connection circuits as shown in Figure 3, five decoupling capacitors are used to improve the ability of anti-jamming.

Fig.3. RS232 level conversion circuit diagram

\section{LCD DRIVE CIRCUIT}

Using LCD display screen in the module, you need to use compatible with the drive circuit,characterized by small size, adding another driver chip, The $74 \mathrm{HC} 245$ chip in as
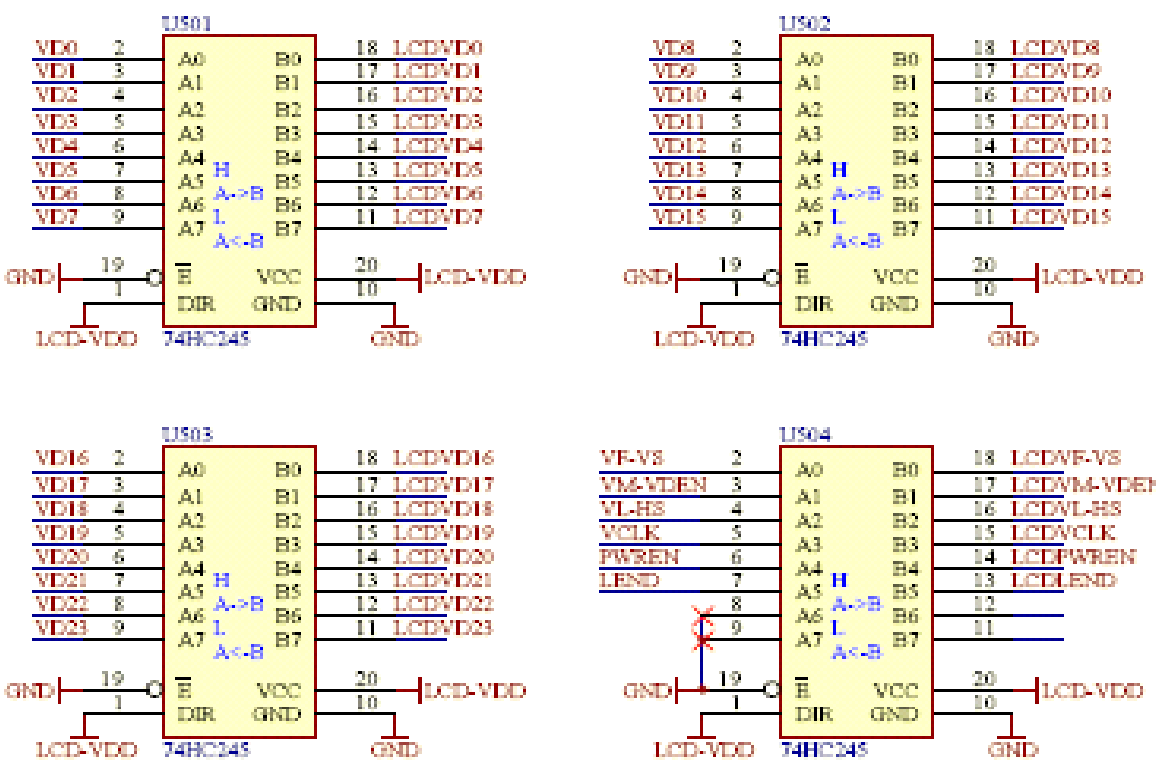

Fig.4. LCD drive circuit diagram

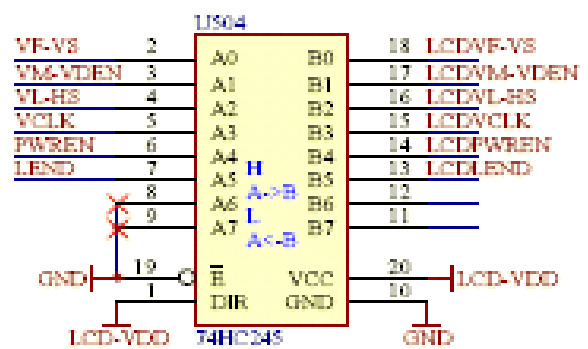

shown in Figure 4. It is a bus transceiver, integrated CMOS Tri-State buffer gate. LCD power supply voltage of $5 \mathrm{~V}$ or $3.3 \mathrm{~V}$ by jumper JP501 Settings, TFT color LCD display with 640 x 480.
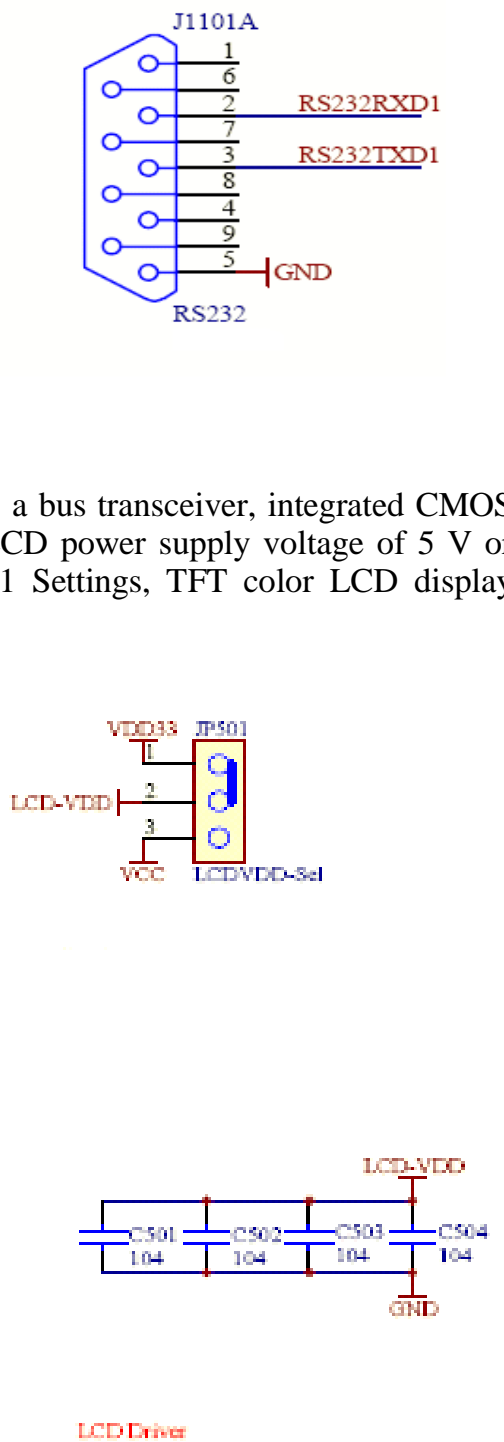


\section{SOFTWARE DESIGN}

Software design mainly involves transplantation of embedded real-time operating system

$\mu \mathrm{C} / \mathrm{OS}$ ii and design of related tasks.

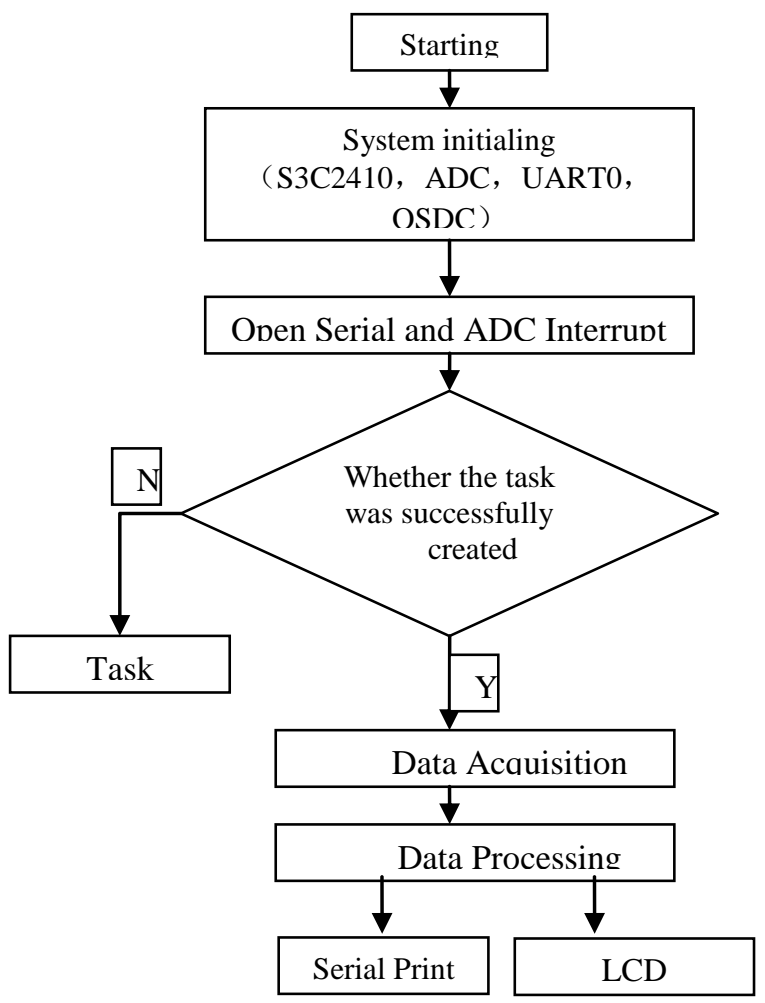

Fig.5. Program flow diagram

\section{TRANSPLANTATION MC/OS ii ON THE S3C2410}

In order to meet the system for real-time and safety requirements, which uses the embedded real-time operating system $\mu$ C/OS-II. It is a priority, multitask preemptive cure real-time kernel

supporting 64 Tasks. The system can support up to 56 user tasks at most,which the idle task priority is 63 and statistical task priority is 64 . First of all, the $\mu$ C/OS-II transplanted to $\mathrm{S} 3 \mathrm{C} 2410$ processor, three related files(OS_CPU.H, OS_CPU_C.C, OS_CPU_A.S) to be modified with the ARM architecture. For OS_CPU. H file, according to the requirements of the emulator unit, modify the data type definition, stack stack growth direction and the macro definition; For OS_CPU_C.C file, 6 operating system function written in C language, namely: OSTaskStkInit(), OSTaskCreateHook(), OSTaskDelHook(), OSTaskSwHook(), OSTaskStatHook(), OSTimeTickHook(). Without special requirements after five functions, they can simply be implement as empty function. For OS_CPU_A.S file, 4 processor-specific functions are written in Assembly language, including OSStartHighRdy(), OS_TASK_SW(), OSIntCtxSw(), OSTickISR(). Mainly data type definition of the compiler, the stack type definitions, macro definitions, and associated with the operating system scheduler function of the preparation.

\section{Subroutine DESIGN}

Tasks include the system initialization process, and serial port initialization handlers, initializing the system messages, initializes the device context, LCD display program, A/D data collection and processing procedures, and so on. After system initialization, data collection procedures to start $\mathrm{A} / \mathrm{D}$ converter, the data collection stored in NAND Flash and transmit into the display buffer. Display program is responsible for buffering the data for display, and serial port handler is responsible for printing the current voltage value.Program flow chart shown in Figure 5. LCD display is primarily through task priority level to adjust the task to achieve. Sine waveform display mainly by creating a device context (DC), namely CreateDC(). ADC device is initialized by init_ADevice() function, which is implemented in the Main_TASKS. The priority of the Main_TASKS is greater than the priority of the AD_Task, where using GetADresult() saves the collected data shown in Figure 6. 


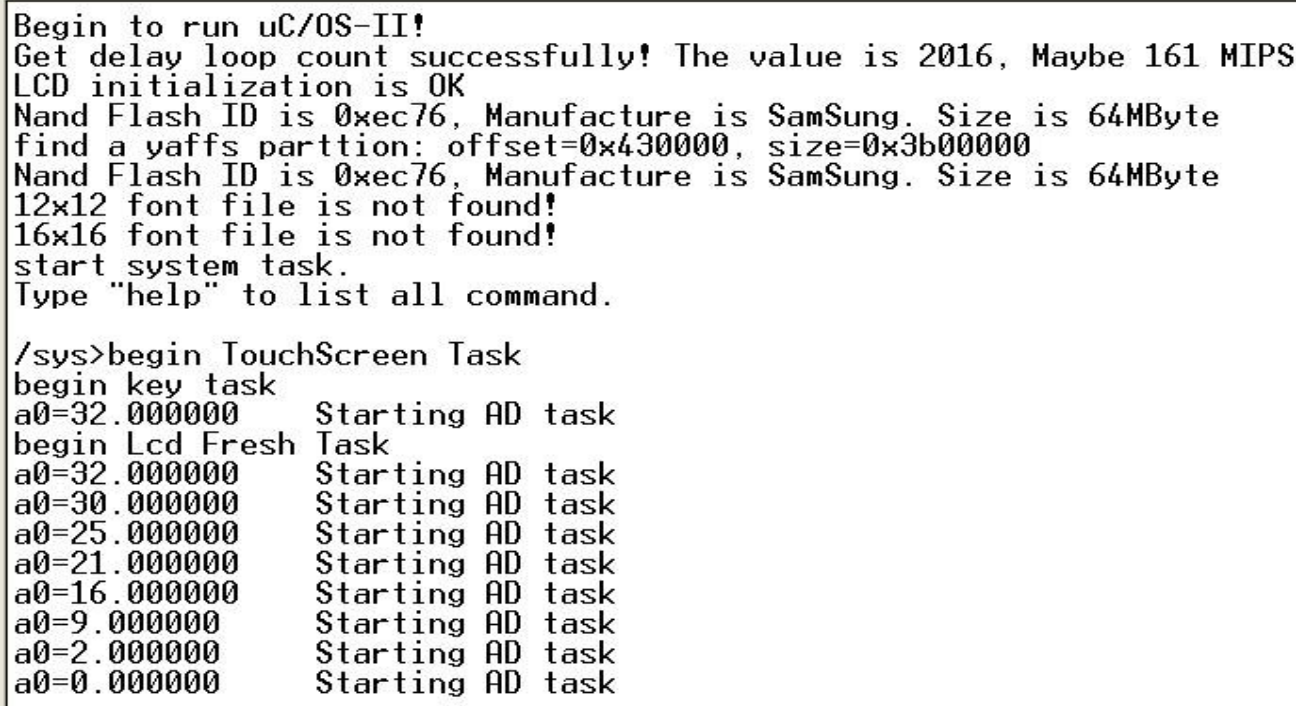

Fig.6. Serial print diagram

\section{CONCLUSIONS}

In this design, data acquisition system based on S3C2410 Microcontroller has completed. System is at the core of 32bit ARM902T Kernel, making full use of existing hardware resources, with its own $\mathrm{ADC}$ as an analog to digital converter, which can improve the conversion efficiency and practicability of the system. In software resource, transplantation $\mu \mathrm{C} / \mathrm{OS}$-II kernel makes the system to easily build and expand, which reliability of system has been greatly improved and has a good application prospect.

\section{References}

[1] Ping Chen, Binlin OuYang. Design of Data Acquisition System Based on ARM [J]. Journal of Northeast Agricultural University, 2008, (4): 99-101.
[2] Li Li, Luyi Xia. Design of Data Acquisition Device Based on ARM Processor and Embedded Real-time Operating Systems [D]. Taiyuan: Taiyuan Polytechnic University, 2006.

[3] Ligong Zhou etc. The ARM Embedded System Fundamental Tutorial [M]. Beijing: Beijing University of Aeronautics and Astronautics Press, 2005.

[4] Qibing Jin. Single-chip Implementation of Data Acquisition Cards and Real-time Multitask Mechanism [J]. Automated Instrumentation, 2002, 23 (6): 33-35.

[5] Rui Liu, Lin Wang. The Design of the Data Acquisition System Based on ARM [J]. China testing, 2010, 4 (4) : 89-92.

[6] Mingjun Tang, Runxian Yang. Design of Embedded Data Acquisition System[J].

[7] Instrumentation and Measurement Technology, 2009,28 (10): 100102. 